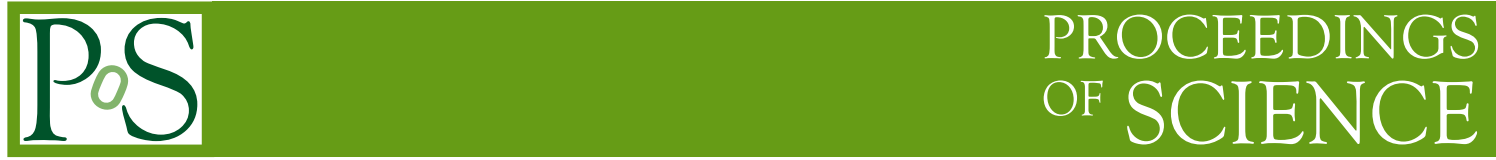

\title{
Flavor Hierarchy in the Confinement Transition of QCD
}

\section{Paolo Alba, Wanda Alberico, Marcus Bluhm* and Claudia Ratti}

Dipartimento di Fisica, Università degli Studi di Torino \& INFN, Sezione di Torino via Giuria 1, I-10125 Torino, Italy

E-mail: paoloalbagdamail.com, albericodto.infn.it, mbluhmete.infn.it,

rattiate.infn.it

\section{René Bellwied}

University of Houston

Houston, TX 77204, USA

E-mail: bellwiedduh.edu

We discuss an experimental test of a possible flavor-hierarchy in the confinement transition of QCD based on quark-number susceptibilities. These fluctuation observables are sensitive to a flavor-specific freeze-out behavior. In this work, we concentrate on the study of the strange-quark sector and investigate the influence of acceptance cuts on our results.

8th International Workshop on Critical Point and Onset of Deconfinement March 11-15

Napa, California, USA

\footnotetext{
* Speaker.
} 


\section{Introduction}

The confinement/deconfinement transition of QCD matter separates the Quark-Gluon Plasma (QGP) phase at large temperatures $T$, where colored quarks and gluons roam quasi-free, from a phase at lower $T$, in which the partons are confined within color-neutral and massive hadrons and resonances. At vanishing baryo-chemical potential $\mu_{B}$, this transition is known to be an analytic crossover [U] taking place over a broad $T$-interval. Depending on the considered transitioncharacterizing quantity, a pseudo-critical temperature $T_{c}$ associated with this phase transformation may be defined. According values for $T_{c}$ were determined in first-principle lattice QCD calculations as $T_{c}=(155 \pm 6) \mathrm{MeV}$ in [园] and $T_{c}=(154 \pm 9) \mathrm{MeV}$ in [3] .

In the ultra-relativistic heavy-ion collisions conducted at LHC and RHIC, the QGP is momentarily produced. For a phenomenological description of the bulk matter emerging from the created fireball, it appears favorable to assume that the chemical freeze-out, in which the chemical composition of the hadronic matter is fixed, takes place immediately after confinement [四]. Accordingly, any detail in the confinement transition should manifest itself in observables that are sensitive to the chemical freeze-out.

Recently, based on lattice QCD calculations of higher-order conserved-charge susceptibilities in the continuum-limit with physical quark masses, the existence of a flavor hierarchy in the confinement transition of QCD was proposed [可, see also [焑]. The idea as such is not new. For example, in [ [ $]$ it was argued within a hydrodynamic analysis of particle spectra measured at RHIC, that hadrons composed of light (up and down) quarks freeze-out at a temperature of about $150 \mathrm{MeV}$, while strange baryons favor a higher freeze-out temperature. In [5] instead (see also [ [8]), combinations of particular conserved-charge susceptibilities were considered, which are stricktly zero in the hadronic phase, while being non-zero once quark degrees of freedom of a specific flavor become liberated. In this way, the onset of deconfinement can be pinned down nonambiguously from first-principles. The lattice QCD results presented in [5], indeed, provided indications for a flavor-specific transition behavior. Accordingly, strange hadrons freeze-out at a higher temperature than hadrons composed of only light quarks, with a separation in $T$ of about $15 \mathrm{MeV}$.

Fluctuations of conserved charges or correlations among different conserved charges can be quantified in a grand canonical ensemble by susceptibilities. Of particular interest, also from an experimental point of view, are the volume-independent ratios of different higher-order susceptibilities. Such ratios constitute observables, which are sensitive to the microscopic structure of the matter and, thus, provide an excellent signal for the confinement/deconfinement transition. A nonmonotonic behavior as a function of $T$ (at $\mu_{B}=0$ ) in the ratios of quartic to quadratic cumulants of net-quark number and electric charge fluctuations, for example, was suggested in [9] as an indicator for the transition. Moreover, susceptibility ratios can be related to higher-order moments of multiplicity distributions [ए]] and are, therefore, in principle measurable in a heavy-ion collision.

In this proceeding, we want to discuss the possibility of experimentally testing a flavor-specific behavior in the confinement transition based on such fluctuation observables. For this purpose, we consider the ratios of quartic to quadratic quark-number susceptibilities $\chi_{4}^{f} / \chi_{2}^{f}$ of flavor $f$, for which continuum-extrapolated lattice QCD results were presented in [可]. These ratios may be related to higher-order moments of particle-identified multiplicity distributions, which are sensitive to the flavor-content and, thus, to a possible flavor-specific chemical freeze-out. 

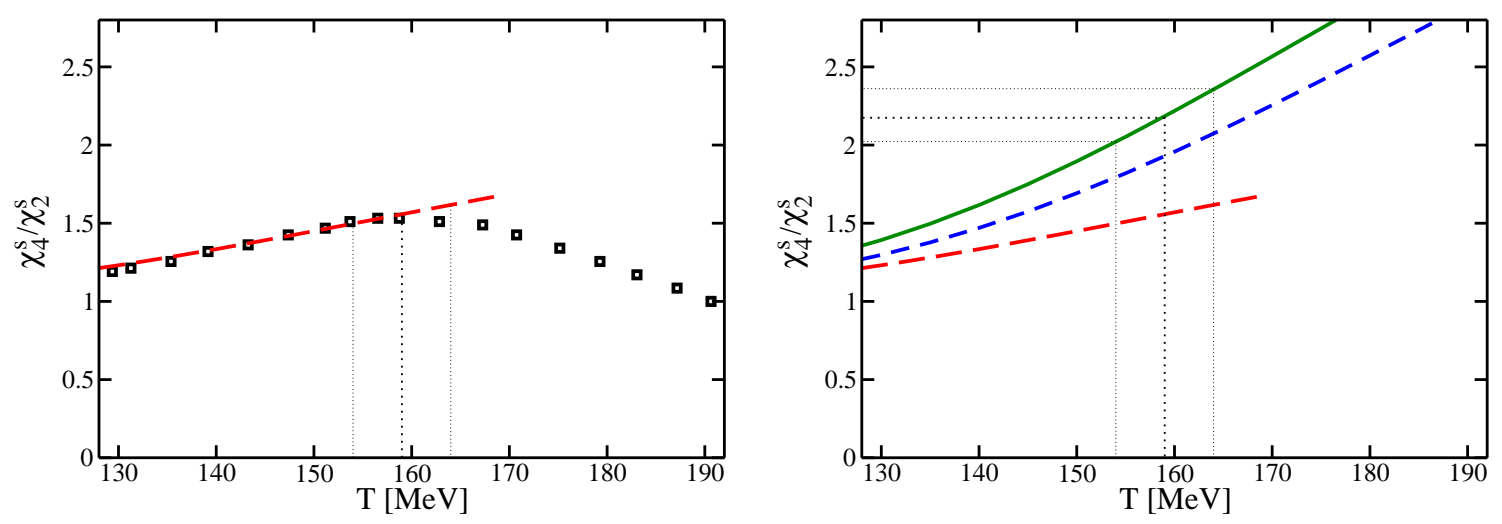

Figure 1: Left: Comparison of HRG model result (long-dashed curve) for $\chi_{4}^{s} / \chi_{2}^{s}$ as function of $T$ with the continuum-extrapolated lattice QCD result (squares) from [ [0]. The used HRG model contains hadron and resonance states up to a mass of $2 \mathrm{GeV}$. Right: The long-dashed curve depicts the HRG model result from the left panel, while the short-dashed curve shows $\chi_{4}^{s} / \chi_{2}^{s}$ for a subset composed of primary $K^{+}, \Lambda^{0}, \Xi^{-}$and $\Omega^{-}$(and their anti-particles). The solid curve highlights the influence of detector acceptance cuts on the latter result (see text for details). The dotted band indicates schematically the possible outcome of a higherorder moment analysis of corresponding multiplicity-distributions, from which the freeze-out temperature of strange quarks could be deduced. Here, the feed-down from strong resonance decays has been neglected.

\section{Toward measuring flavor-specific fluctuation observables}

In our attempt of defining a measurement suitable for testing the flavor hierarchy, we focus on the physical situation met at the LHC and neglect finite $\mu_{B}$-effects in our considerations. ALICE has measurements of identified $\pi, K, p, \Lambda, \Xi$ and $\Omega$ at their disposal. By measuring hadronic multiplicity distributions, one may aim at determining the freeze-out behavior of a specific quarkflavor from a higher-order moment analysis and the comparison of the associated light and strange quark-number susceptibility ratios. In the following, we will concentrate on the quark-number fluctuations in the strange-quark sector, only. The situation in the light-quark sector is more involved due to the influence of pions, which need to be taken into account properly.

For an interpretation of the measured data and an extraction of the relevant physics, a comparison with phenomenological models is often useful. The hadron resonance gas (HRG) model is known to provide a suitable tool for the description of the thermodynamics of QCD in the hadronic phase. Details of the model are described, for example, in [ए]]. A comparison of the HRG model result for $\chi_{4}^{s} / \chi_{2}^{s}$ (including hadrons and resonances up to a mass of $2 \mathrm{GeV}$ ) with the corresponding lattice QCD result from [5] shows an impressive agreement in a certain $T$-interval, cf. Fig. $($ (left panel). The onset of a deviation of the HRG model from the lattice QCD data may be interpreted as a rough indicator for the initiating liberation of strange quarks from their confining hadrons. Although for a more precise estimate the influence of resonance states heavier than $2 \mathrm{GeV}$ should be investigated, the change in the trend of $\chi_{4}^{s} / \chi_{2}^{s}$ clearly signals a change in the relevant degrees of freedom.

As a strange-quark specific measurement, we consider a subset composed of primary $K^{+}, \Lambda^{0}$, $\Xi^{-}$and $\Omega^{-}$as well as their anti-particles. The short-dashed curve in Fig. $\square$ (right panel) shows the corresponding $\chi_{4}^{s} / \chi_{2}^{s}$-result for this subset. By analyzing the higher-order moments of the 
combined flavor-content sensitive multiplicity-distributions, one could, in principle, determine the freeze-out temperature for strange quarks. This strategy is highlighted by the dotted band in Fig. W (right panel).

An accurate comparison, however, requires the consideration of several aspects. Apart from the necessity of an efficiency correction of the measured data, applied detector acceptance cuts have to be taken into account for a meaningful model-interpretation. This can be achieved by rewriting the momentum-integrals in the partition function of the HRG model (see [ए]]) in terms of transverse momentum $p_{T}$ and pseudo-rapidity $\eta$. The influence of acceptance cuts in $p_{T}$ and $\eta$, e.g. $0.2<p_{T}(\mathrm{GeV} / \mathrm{c})<2.0$ and $|\eta|<0.5$, on the result of the considered subset is depicted by the solid curve in Fig. $\square$ (right panel). Moreover, strong resonance decays influence the outcome of such an analysis. The impact of these decays on the fluctuation observables will be reported in a forthcoming publication.

\section{Acknowledgments}

The work of C. Ratti and M. Bluhm is supported by funds provided by the Italian Ministry of Education, Universities and Research under the Firb Research Grant RBFR0814TT.

\section{References}

[1] Y. Aoki, G. Endrodi, Z. Fodor, S. D. Katz, and K. K. Szabo, The order of the quantum chromodynamics transition predicted by the standard model of particle physics, Nature 443 (2006) 675.

[2] S. Borsanyi et al., Is there still any $T_{c}$ mystery in lattice QCD? Results with physical masses in the continuum limit III, J. High Energy Phys. 1009 (2010) 073.

[3] A. Bazavov et al., The chiral and deconfinement aspects of the QCD transition, Phys. Rev. D 85 (2012) 054503.

[4] U. Heinz and G. Kestin, Universal chemical freeze-out as a phase transition signature, in proceedings of CPOD 2006, POS (CPOD2006) 038 .

[5] R. Bellwied, S. Borsanyi, Z. Fodor, S. D. Katz, and C. Ratti, Is there a flavor hierarchy in the deconfinement transition of QCD?, arXiv:1305.6297[hep-lat].

[6] C. Ratti, R. Bellwied, M. Christoforetti, and M. Barbaro, Are there hadronic bound states above the QCD transition temperature?, Phys. Rev. D 85 (2012) 014004.

[7] P. Huovinen, Chemical freeze-out temperature in the hydrodynamical description of Au+Au collisions at $\sqrt{s_{N N}}=200 \mathrm{GeV}$, Eur. Phys. J. A37 (2008) 121.

[8] A. Bazavov et al., Strangeness at high temperatures: from hadrons to quarks, arXiv:1304.7220 [hep-lat].

[9] S. Ejiri, F. Karsch, and K. Redlich, Hadronic fluctuations at the QCD phase transition, Phys. Lett. B633 (2006) 275.

[10] F. Karsch and K. Redlich, Probing freeze-out conditions in heavy ion collisions with moments of charge fluctuations, Phys. Lett. B695 (2011) 136.

[11] S. Borsanyi et al., The QCD equation of state with dynamical quarks, J. High Energy Phys. 1011 (2010) 077. 